Numerical and experimental study on the steady cone-jet mode of electro-centrifugal spinning

Ali Reza Hashemi, Ahmad Reza Pishevar, Afsaneh Valipouri, and Emilian I. Părău

Citation: Physics of Fluids 30, 017103 (2018);

View online: https://doi.org/10.1063/1.5001808

View Table of Contents: http://aip.scitation.org/toc/phf/30/1

Published by the American Institute of Physics

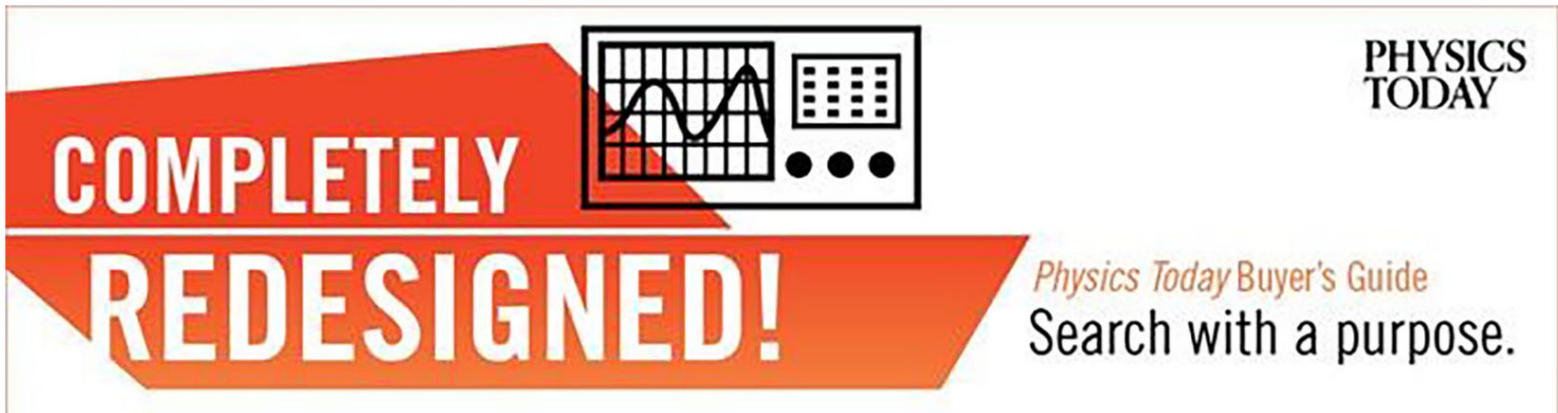




\title{
Numerical and experimental study on the steady cone-jet mode of electro-centrifugal spinning
}

\author{
Ali Reza Hashemi, ${ }^{1}$ Ahmad Reza Pishevar, ${ }^{1, a)}$ Afsaneh Valipouri, ${ }^{2}$ and Emilian I. Părău ${ }^{3}$ \\ ${ }^{1}$ Department of Mechanical Engineering, Isfahan University of Technology, Isfahan 84156-83111, Iran \\ ${ }^{2}$ Department of Textile Engineering, Isfahan University of Technology, Isfahan 84156-83111, Iran \\ ${ }^{3}$ School of Mathematics, University of East Anglia, Norwich NR4 7TJ, United Kingdom
}

(Received 27 August 2017; accepted 16 December 2017; published online 8 January 2018)

\begin{abstract}
This study focuses on a numerical investigation of an initial stable jet through the air-sealed electrocentrifugal spinning process, which is known as a viable method for the mass production of nanofibers. A liquid jet undergoing electric and centrifugal forces, as well as other forces, first travels in a stable trajectory and then goes through an unstable curled path to the collector. In numerical modeling, hydrodynamic equations have been solved using the perturbation method - and the boundary integral method has been implemented to efficiently solve the electric potential equation. Hydrodynamic equations have been coupled with the electric field using stress boundary conditions at the fluid-fluid interface. Perturbation equations were discretized by a second order finite difference method, and the Newton method was implemented to solve the discretized non-linear system. Also, the boundary element method was utilized to solve electrostatic equations. In the theoretical study, the fluid was described as a leaky dielectric with charges only on the surface of the jet traveling in dielectric air. The effect of the electric field induced around the nozzle tip on the jet instability and trajectory deviation was also experimentally studied through plate-plate geometry as well as point-plate geometry. It was numerically found that the centrifugal force prevails on electric force by increasing the rotational speed. Therefore, the alteration of the applied voltage does not significantly affect the jet thinning profile or the jet trajectory. Published by AIP Publishing. https://doi.org/10.1063/1.5001808
\end{abstract}

\section{INTRODUCTION}

Fibers with diameters of $100-500 \mathrm{~nm}$, known as nanofibers, have attracted considerable attention in the last decade-owing to their unique characteristics and potential applications with regard to medical textiles, filtration, protective textiles, electronics, and optics. ${ }^{1}$ So far, many different techniques such as force-spinning, ${ }^{2}$ electro-spinning,,${ }^{3,4}$ melt blowing, ${ }^{5}$ phase separation, ${ }^{6}$ self-assembly, ${ }^{7}$ and template synthesis ${ }^{8,9}$ have been introduced for the fabrication of polymer nanofibers. Among them, electro-spinning is a well-established and intensively investigated methodology, and it appears to be the only known technique that is suitable to fabricate continuous nanofibers. However, a major shortcoming of electro-spinning is the low production rate compared to conventional fiber spinning. Heretofore, several efforts have been made to increase the production rate of nanofibers.

One of the mass production methods of nanofibers is to utilize centrifugal force rather than electric force (as in electrospinning) or to combine centrifugal and electric forces simultaneously. Force-spinning, ${ }^{10}$ electro-centrifugal spinning, ${ }^{11}$ air-sealed centrifuge spinning, ${ }^{1}$ and air-sealed electrocentrifugal spinning ${ }^{12}$ all have proven successful as viable methods to mass-produce polymeric nanofibers, allowing a significant increase in yield and simplifying the production. In

a) Author to whom correspondence should be addressed: apishe@cc.iut.ac.ir centrifuge spinning, a liquid jet undergoes centrifugal force, Coriolis force, viscous effects, and air drag. ${ }^{2}$ Coriolis and drag forces serve to bend up the jet trajectory. In fact, air resistance causes the liquid jet to deflect, progressively.

When the liquid jet is exposed to the high-speed airflow, the jet loses its solvent rapidly. Therefore, it becomes more difficult to elongate the jet, which leads to thicker nanofibers. ${ }^{1}$ Recently published work has presented the air-sealed electro-centrifugal spinning as a mass production method of nanofibers which eliminates the air resistance in centrifuge spinning. ${ }^{12}$ In current work, air-sealed electro-centrifugal spinning has been used for experimental purposes.

Studies on the irrotational curved liquid sheets and jets have been done by Vanden-Broeck and Keller, ${ }^{13}$ Entov and Yarin, ${ }^{14}$ Dias and Vanden-Broeck, ${ }^{15}$ Finnicum et al. ${ }^{16}$ Cummings and Howell, ${ }^{17}$ Hohman et al., ${ }^{3}$ and Reneker and Yarin. ${ }^{18}$

Wallwork et al., ${ }^{19}$ Decent et al., ${ }^{20}$ Wong et al., ${ }^{21}$ and Părău et $a .^{22}$ experimentally and numerically investigated the prilling process for the production of fertilizers, considering a rotational jet.

Dabirian et $a l . .^{23-25}$ have experimentally investigated electro-centrifugal spinning. They found that increasing the electrical voltage has no effect on the nanofiber diameter, and the diameter of the nanofibers increases with increasing rotational speed. Also, a proper adjustment of the solution concentration, applied electric voltage, and rotational speed causes a significant increase in the production rate of 
nanofibers-rather than that of the electro-spinning process. Valipouri et al. ${ }^{1}$ presented a numerical and experimental investigation on liquid jet dynamics through air-sealed centrifuge spinning. They observed fairly good agreement between the isolated jet trajectory and the model-predicted one. Also, the liquid jet trajectory through air-sealed centrifuge spinning as well as non-isolated centrifuge spinning was experimentally studied; and it was found that the non-isolated jet curved more compared to the others, which is due to air drag.

The present study focuses on investigating the behavior of an incompressible Newtonian leaky dielectric jet through air-sealed electro-centrifugal spinning. We considered only the steady stretching process of the liquid jet. Electrohydrodynamic (EHD) equations were derived using the perturbation method. The electrostatic equation-which is the Laplace equation-was solved using the boundary integral technique, as conducted by Lac and Homsy. ${ }^{26}$ In order to describe the dynamics of electric charge transport at the fluidfluid interface, we used the charge conservation equation as proposed by Saville. ${ }^{27}$ The main essential transport mechanisms in this equation are as follows: the charge accumulation at the interface due to conduction and the jump in the conventional charge current in the bulk across the interface; surface convection of the interfacial charges; the effect of stretching of the interface; and surface conduction. In the present study, due to low flow rates and high voltages, the charge convection term can be eliminated from the governing equation. Moreover, by assuming an instantaneous migration of electric charges toward the interface, a concentration change due to the surface dilation in the transport of electric charges is negligible, and the electric current across the interface will become continuous. Hence, the surface charge conservation equation is reduced to a boundary condition at the interface. This model, which was well adopted for the leaky dielectric systems, is called the static model for surface charges. Moreover, the proposed numerical method to solve the governing electrical equation is capable of mimicking the effect of the nozzle in the applied electric field without considering its geometry. Subsequently, the resulting irregularity around the nozzle will be automatically compensated. In addition, the effect of unevenness of the electric field around the nozzle tip was experimentally studied through plate-plate geometry as well as point-plate geometry. It was observed that connecting the high voltage source to the nozzle will result in more irregularity in the electric field around the nozzle. This irregularity causes instabilities and elongates the jet in a shorter distance from the nozzle at the low applied electric field. For the sake of validation, current numerical results were compared to experimental results.

\section{A. Spinning process}

The spinning process was carried out through air-sealed electro-centrifugal spinning, introduced as follows (Fig. 1). A syringe filled with a solution was held close to a rotating cylindrical receptacle. A nozzle attached to the syringe was electrified by applying an electric field between the nozzle and the cylindrical collector. When the rotational speed of the rotary head and the electric potential difference increase enough to exceed the surface tension, a fluid jet ejects from the tip of the nozzle. The electric force and the centrifugal force stretch the jet, making it very fine. The jet is collected in the form of a nanofiber mat on the metallic cylindrical collector.

\section{GOVERNING EQUATIONS}

The equation of continuity and momentum is applied to an incompressible Newtonian fluid as

$$
\begin{aligned}
\boldsymbol{\nabla} \cdot \boldsymbol{u}= & 0, \\
\rho\left(\frac{\partial \boldsymbol{u}}{\partial t}+(\boldsymbol{u} \cdot \boldsymbol{\nabla}) \boldsymbol{u}\right)= & -\boldsymbol{\nabla} p+\boldsymbol{\nabla} \cdot \boldsymbol{\tau}-2 \omega \times \boldsymbol{u}-\boldsymbol{\omega} \\
& \times(\boldsymbol{\omega} \times \boldsymbol{r})+\rho \boldsymbol{g},
\end{aligned}
$$

where $\boldsymbol{u}$ is the velocity; $\rho$ is the density; $p$ is the pressure; $\tau$ is the deviatoric stress tensor, which is composed of two hydrodynamic $\left(\tau^{H}\right)$ and electric $\left(\tau^{E}\right)$ components; $\boldsymbol{\omega}$ is the

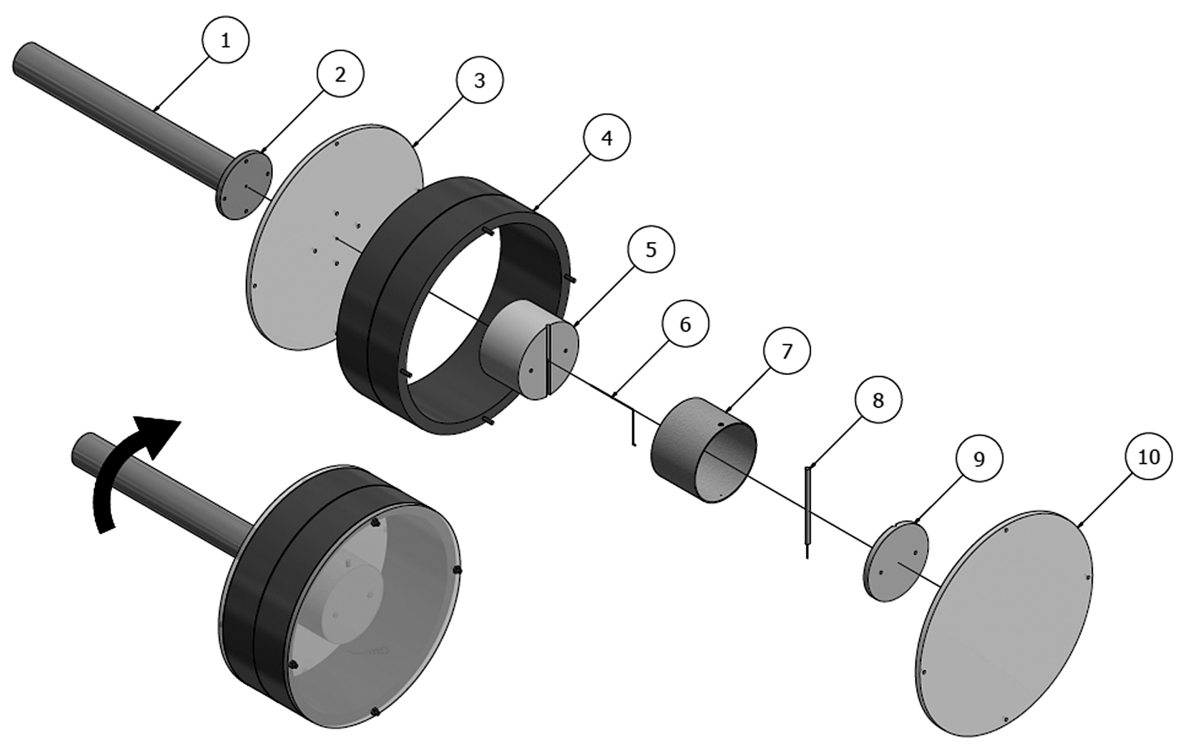

FIG. 1. The air-sealed electrocentrifugal spinning setup which proposed by Valipouri et al. ${ }^{1}$ Setup components are shaft (1), flange (2), back transparent plastic cover (3), metal collector (4), internal rotary plastic cylinder (5), wire (6), internal rotating cylinder cladding (7), needle and syringe (8), internal rotary plastic cylinder cover (9), and front transparent plastic cover (10). Poles of high voltage source are connecting to shaft (1) and metal collector (4) by sliding mechanism which slides in a groove on the external surface of the metal collector, respectively. This is point-plate geometry if part No. 7 is eliminated; otherwise, this is plate-plate spinning geometry. 
angular velocity; $\boldsymbol{r}$ is the position vector on the jet surface; and $\boldsymbol{g}$ is the gravitational acceleration vector. For a leaky dielectric fluid, electric force is only applied on the fluidfluid interface. Hence, the electric force will be a boundary term, and the induced current will be formed only by the application of boundary conditions on the interface. ${ }^{28}$ Saville ${ }^{27}$ determined the jump condition of normal and tangential Maxwell electric stresses at the interface as presented in Eqs. (3) and (4),

$$
\begin{aligned}
\boldsymbol{n} \cdot\left\|\boldsymbol{\tau}^{E}\right\| \cdot \boldsymbol{n}^{T} & =\frac{\varepsilon_{0}}{2}\left\|\varepsilon\left((\boldsymbol{E} \cdot \boldsymbol{n})^{2}-(\boldsymbol{E} \cdot \boldsymbol{t})^{2}\right)\right\|, \\
\boldsymbol{t} \cdot\left\|\boldsymbol{\tau}^{E}\right\| \cdot \boldsymbol{n}^{T} & =q^{s}(\boldsymbol{E} \cdot \boldsymbol{t}) .
\end{aligned}
$$

In these equations, $\|(\cdot)\|$ denotes the jump, outside-inside, of $(\cdot)$ across the interface; $\varepsilon_{0} \varepsilon$ indicates the electric permittivity of the fluid; $q^{s}$ is the electric charge surface density; and $\boldsymbol{n}$ and $\boldsymbol{t}$ are the unit normal and the tangential vectors to the free surface, respectively. In this study, electric charges were modeled as static, and the electric charge density was obtained explicitly from the equation $q^{s}=\varepsilon_{0}\|\varepsilon \boldsymbol{E} \cdot \boldsymbol{n}\|{ }^{26,27}$ Across the jet interface, $\|\boldsymbol{E} \cdot \boldsymbol{t}\|$ is continuous but $\|\boldsymbol{E} \cdot \boldsymbol{n}\|$ undergoes a discontinuity due to the difference of physical properties of the two fluids. ${ }^{26}$ In order to consider electric stresses in the momentum equation, the jump condition was applied across the interface. Additionally, surface tension results in pressure discontinuity across the interface. The pressure jump on the interface is directly proportional to the average of surface local curvature, causing a difference in pressure between the inside and outside of the jet. With regard to these two effects, the jump condition in normal stresses can be calculated as in Eq. (5), ${ }^{29}$

$$
\left\|\left(\begin{array}{c}
\boldsymbol{n} \\
\boldsymbol{t}
\end{array}\right) \cdot(p \boldsymbol{I}-\boldsymbol{\tau}) \cdot \boldsymbol{n}^{T}\right\|=\left(\begin{array}{c}
\gamma \kappa \\
0
\end{array}\right),
$$

where $\gamma$ is the surface tension, $\kappa$ is the surface curvature, and $\boldsymbol{I}$ is the unit tensor. The dimensionless parameters were listed in Table I, where $U$ is the velocity of the jet at the nozzle outlet; $a$ is the radius of the nozzle; $R$ is the jet local radius; $X, Y$, and $Z$ are the jet axis coordinates; and $E_{n, t}$ are the normal and the tangential electrical components on the interface. The dimensionless numbers in this study include Webber, Reynolds, Froude, Rossby, Beta, aspect ratio, electric permittivity ratio, and conductivity ratio-as listed in Table II, where $\mu$ is the dynamic viscosity, the magnitude of the external electric field is $E_{0}, \varepsilon_{0} \varepsilon_{o}$ is the electric permittivity of the external fluid, the distance between the nozzle tip and the center of the rotating cylinders is $s_{0}$, and $\omega$ is the angular speed.

The basis used for solving the equations governing the electrified jet through air-sealed electro-centrifugal spinning is the perturbation theory proposed by Păău et al., ${ }^{22}$ who used this methodology to simulate the behavior of a bent jet

TABLE I. Dimensionless parameters.

\begin{tabular}{lcccc}
\hline \hline $\bar{u}=\frac{u}{U}$ & $\bar{t}=\frac{t U}{a}$ & $\bar{p}=\frac{p}{\rho U^{2}}$ & $\bar{q}^{s}=\frac{q^{s}}{\varepsilon_{0} \varepsilon_{0}}$ & $\bar{E}_{n, t}=\frac{E_{n, t}}{E_{0}}$ \\
\hline $\bar{R}=\frac{R}{a}$ & $\bar{s}=\frac{s}{a}$ & $\bar{X}=\frac{X}{a}$ & $\bar{Y}=\frac{Y}{a}$ & $\bar{Z}=\frac{Z}{a}$ \\
\hline \hline
\end{tabular}

TABLE II. Dimensionless numbers.

\begin{tabular}{llll}
\hline \hline$W e=\frac{\rho U^{2} a}{\gamma}$ & $R e=\frac{\rho U a}{\mu}$ & $F r=\frac{U^{2}}{a g}$ & $R b=\frac{U}{a \omega}$ \\
\hline$\epsilon=\frac{a}{s_{0}}$ & $\beta=\frac{\varepsilon_{0} \varepsilon_{o} E_{0}^{2}}{\rho U^{2}}$ & $\varepsilon_{i o}=\frac{\varepsilon_{i}}{\varepsilon_{o}}$ & $\sigma_{o i}=\frac{\sigma_{o}}{\sigma_{i}}$ \\
\hline \hline
\end{tabular}

in a prilling process. They expanded the velocity components, pressure, radius, and position components of the jet trajectory in an asymptotic series by assuming that the jet is a long, slender object. They then substituted these expansions in continuity and momentum equations as well as boundary conditions and, after a few manipulations, obtained the governing equations of the jet behavior. Specific assumptions such as circular cross section for the jet were consideredand the position of the centerline was not affected by the small perturbations. Their boundary conditions included the jump in the pressure magnitude (5) and kinematic boundary conditions on the surface. In the present study, only the stable region was investigated. Hence, time-dependent terms could be eliminated, and the governing perturbation equations for the electro-hydrodynamic behavior of the axisymmetric jet correspond to Eqs. (6)-(9), with known electric field components,

$$
\begin{gathered}
\bar{u} \bar{u}_{s}=-\bar{p}_{s}+\frac{1}{R e}\left(2 \bar{u}_{s s}+\frac{6 \bar{R}_{s} \bar{u}_{s}}{\bar{R}}\right)+\beta \frac{2\left(1-\varepsilon_{i o} \sigma_{o i}\right) \bar{E}_{o_{n}} \bar{E}_{o_{t}}}{\bar{R}} \\
+\frac{1}{R b^{2}}\left(\left(\bar{X}+\frac{1}{\epsilon}\right) \bar{X}_{s}+\bar{Z} \bar{Z}_{s}\right) \\
-\left(\bar{X}_{s} \bar{Z}_{s s}-\bar{X}_{s s} \bar{Z}_{s}\right)\left(\bar{u}^{2}-\frac{1}{W e \bar{R}}-\frac{3}{R e} \bar{u}_{s}\right)-\frac{2 \bar{u}}{R b} \\
+\frac{1}{R b^{2}}\left(\left(\bar{X}+\frac{1}{\epsilon}\right) \bar{Z}_{s}-\bar{X}_{s} \bar{Z}\right)=0 \\
\bar{X}_{s}^{2}+\bar{Z}_{s}^{2}=1 \\
\frac{\left(\bar{u} \bar{R}^{2}\right)_{s}}{2 \bar{R}}=0
\end{gathered}
$$

The applied coordinate system is an extension of the cylindrical polar coordinates, $(s, n, \varphi)$, where $s$ is the arclength along the jet axis and $(n, \varphi)$ is the polar coordinate in any cross section of the jet. The centerline was described by a Cartesian coordinate system $(X(s, t), 0, Z(s, t))$. This system was established and is shown in more detail in Fig. 2.

The origin of this Cartesian coordinate system is the center of the nozzle outlet, and the jet position vector can be written as $\bar{r}_{c l}=\bar{X} \boldsymbol{e}_{i}+\bar{Z} \boldsymbol{e}_{k}$, where $\boldsymbol{e}_{i}$ and $\boldsymbol{e}_{k}$ are unit vectors in the Cartesian coordination system. The angular velocity is defined as $\omega=\omega \boldsymbol{e}_{j}$.

Equations (6) and (7) are described as momentum equations in axial and radial directions-in which the oi and io indices represent the quantitative ratio of the exterior to interior fluid and vice versa, respectively. As should be expected in a slender jet theory, we assumed that the cross section of the jet remains circular and that the momentum does not depend on $\varphi$ at the leading order. Readers are referred to Părău et al. ${ }^{22}$ for further details regarding the derivation of governing equations and the implementation of a perturbation 


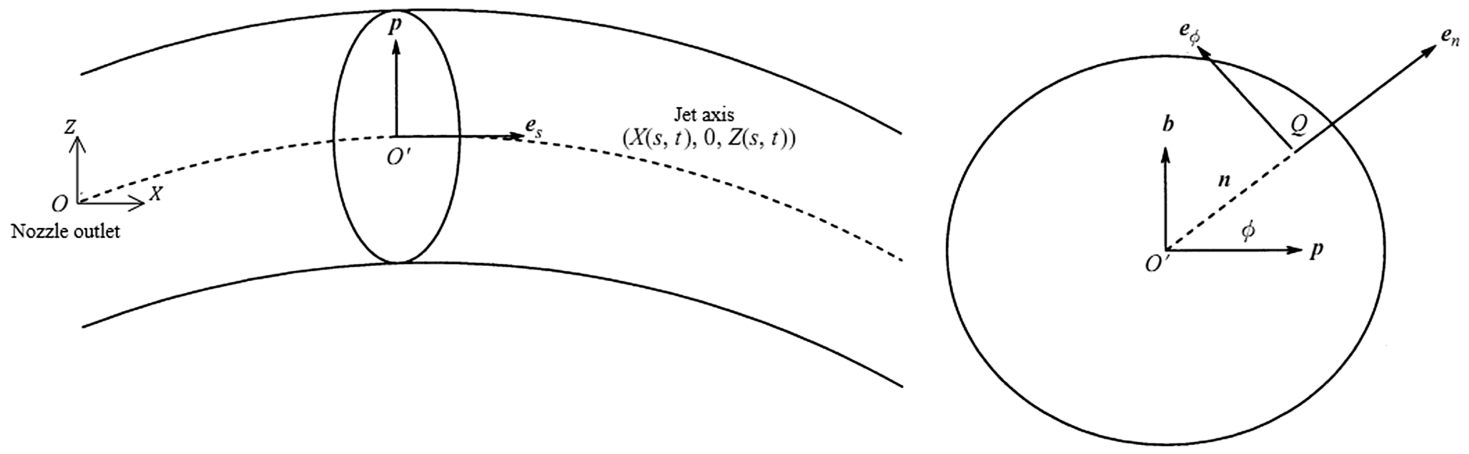

(a)

(b)

FIG. 2. Sketch of a curved jet in the $X-Z$ plane and a cross section of a curved jet.

analysis. According to $d \bar{s}^{2}=d \bar{X}^{2}+d \bar{Z}^{2}$, the arc-length can be obtained from Eq. (8). Equation (9) was obtained based on the kinematic boundary condition. From this equation, it has been observed that $\bar{u} \bar{R}^{2}$ is constant, and by using the boundary condition $\bar{R}(0)=\bar{u}(0)=1$ at the nozzle tip, the axial velocity will be obtained as $\bar{u}=1 / \bar{R}^{2}$. In Eq. (6), the pressure (which is composed of hydrodynamic and electrostatic pressure components) can be calculated by the following equation:

$\bar{p}=\frac{1}{W e \bar{R}}-\frac{\bar{u}_{s}}{R e}-\frac{\beta}{2}\left(\left(1-\varepsilon_{i o} \sigma_{o i}^{2}\right) \bar{E}_{o_{n}}^{2}-\left(1-\varepsilon_{i o}\right) \bar{E}_{t}^{2}\right)$.

In present study, due to the assumption of instantaneous migration of electric charges to the fluid-fluid interface, electric stresses are not included in the radial momentum Eq. (7).

Electrical phenomena are described by Maxwell's electromagnetic equations. However, in the absence of external magnetic fields conditions, if the characteristic time scale of magnetic phenomena $\left(t_{M}\right)$ is much smaller than the characteristic time scale of electric phenomena $\left(t_{c}\right)$, magnetic effects can be ignored completely and the electrostatic equations provide for an accurate approximation. ${ }^{27}$ Moreover, charges in the leaky dielectric fluid only accumulate at the interface, which can be considered as a boundary effect, modifying the external electric field. ${ }^{27,28}$ Therefore, for a leaky dielectric fluid system, the governing equation is reduced to the simple electric current continuity law and can be represented as follows:

$$
\boldsymbol{\nabla} \cdot(\sigma \boldsymbol{E})=0,
$$

where $\sigma$ is the electric conductivity of the fluid. Due to the irrotational property of the electric field, it can be considered as the gradient of an electric potential $\boldsymbol{E}=-\nabla \Psi$ and we have

$$
\boldsymbol{\nabla} \cdot(\sigma \boldsymbol{\nabla} \Psi)=0 .
$$

To complete the description, a charge conservation equation is required at the interface. By ignoring the charge diffusion mechanism, this equation can be written as follows: ${ }^{27}$

$\frac{t_{c}}{t_{P}} \frac{\partial q^{s}}{\partial t}+\frac{t_{c}}{t_{F}}\left(\boldsymbol{u} \cdot \nabla_{s} q^{s}-q^{s} \boldsymbol{n} \cdot(\boldsymbol{n} \cdot \boldsymbol{\nabla}) \boldsymbol{u}\right)=\|-\sigma \boldsymbol{E} \cdot \boldsymbol{n}\|$,

where $t_{c}$ is the electrostatic time scale identified by the ratio of the dielectric permeability and conductivity, $t_{P}$ is the transport process time arising from viscose relaxation and diffusion, $t_{F}$ is the convective flow time, which can be defined as the ratio of the flow length scale to the flow characteristics speed, and $\boldsymbol{\nabla}_{s}$ is the surface gradient. In this equation, the first term on the left represents charge relaxation, the second term describes charge convection at the interface, and the third term denotes changes in concentration due to dilation of the surface. Also, the term on the right stands for charge transport to the surface by electro-migration. For a steady flow motion and the assumption of instantaneous migration of charges to the interface, $t_{c} / t_{F} \ll 1$, Eq. (13) is reduced to the continuity of the electric current at the interface. It is also used as a boundary condition to solve the equation for the electric current equation.

In this study, the physical and electrical properties of the fluids are constant. Hence, the potential distribution (12) can be transformed into a potential Laplace equation which would be solved by a boundary integral approach, similar to the approach of Lac and Homsy. ${ }^{26}$ They stated that the extent of the electric field on the surface is the average of interior and exterior electric fields in the vicinity of the interface. By applying the boundary condition of electric current continuity across the interface, i.e., $\|(\sigma \boldsymbol{E} \cdot \boldsymbol{n}=0)\|$, and by applying the external electric field with the assumption of no free charges on the surface, the dimensionless boundary integral equation of electric field for each of the surface elements can be obtained using Eq. (14),

$$
\left(\frac{\overline{\boldsymbol{E}}_{o}+\overline{\boldsymbol{E}}_{i}}{2}\right)_{p}=\frac{\boldsymbol{E}_{\infty}}{E_{0}}+\frac{1-\sigma_{o i}}{4 \pi} \oint_{(\bar{S})_{q}} \frac{(\overline{\boldsymbol{r}})_{p q}}{(\bar{r})_{p q}^{3}}\left(\bar{E}_{o_{n}}\right)_{q} d(\bar{S})_{q},
$$

where $p, q$, and $(\bar{r})_{p q}$ are field point, source point, and the distance between these two points, $\boldsymbol{E}_{\infty}$ is the applied electric field vector, and $\bar{S}$ is the fluid-fluid interface, respectively. The first term on the right side of this equation represents the applied electric field at each point of the computational domain, and the second term represents the electric field correction at each point due to the existence of a potential surface. This equation includes two variables of $\boldsymbol{E}_{p}$, the electric field vector at each point, and $\left(\bar{E}_{o_{n}}\right)_{q}$, the electric field normal to the surface. Lac and Homsy ${ }^{26}$ primarily solved this equation for each point of the surface, obtained by the dot product of both sides of this equation by the unit normal vector of the field point $(p)$. Using this approach, the electric field normal to the surface in each 
point can be obtained as in Eq. (15),

$$
\begin{aligned}
\frac{1+\sigma_{o i}}{2}\left(\bar{E}_{O_{n}}\right)_{p}= & \frac{\boldsymbol{E}_{\infty}}{E_{0}} \cdot(\boldsymbol{n})_{p} \\
& +\frac{1-\sigma_{o i}}{4 \pi} \oint_{(\bar{S})_{q}} \frac{(\overline{\boldsymbol{r}})_{p q} \cdot(\boldsymbol{n})_{p}}{(\bar{r})_{p q}^{3}}\left(\bar{E}_{o_{n}}\right)_{q} d(\bar{S})_{q} .
\end{aligned}
$$

After the calculation of the normal component of the electric field and its substitution into Eq. (14), an electric field vector will be obtained for surface points. Thereafter, the tangential component of the electric field can be obtained with a vector subtraction, as in Eq. (16),

$$
\left(\bar{E}_{t} \boldsymbol{t}\right)_{p}=\left(\frac{\overline{\boldsymbol{E}}_{o}+\overline{\boldsymbol{E}}_{i}}{2}\right)_{p}-\left(\bar{E}_{o_{n}} \boldsymbol{n}\right)_{p} .
$$

Since the tangential electric field component is continued across the interface, $\bar{E}_{o_{t}}=\bar{E}_{i_{t}}=\bar{E}_{t}$.

\section{NUMERICAL SOLUTION}

In the present study, we analyze the interface behavior of a rotating liquid jet surrounded with air under an external electric field. In order to numerically solve the boundary integral Eq. (15), the boundary element method was implemented. This numerical method required the initial surface in order to perform the calculations. Hence, it was primarily assumed that the electric field does not exist and that the liquid jet leaves the nozzle with the radius of $a$ with a determined flow rate of $Q=U \pi a^{2}$ under the centrifugal force. Under these conditions, the initial surface was obtained, subsequently, and applying the electric field will provide the actual, final jet profile.

The idea of singularity cancellation in a boundary integral equation in a $3 D$ domain was adopted from Kirkup..$^{30} \mathrm{He}$ proposed a correction formula for a Gaussian quadrature over a triangular element to eliminate the singularity of the integrand, Eq. (17),

$$
\boldsymbol{I}=\oint_{(\bar{S})_{q}} \frac{(\overline{\boldsymbol{r}})_{p q} \cdot(\boldsymbol{n})_{p}}{(\bar{r})_{p q}^{3}}\left(\bar{E}_{O_{n}}\right)_{q} d(\bar{S})_{q} .
$$

The coordinate system under consideration with regard to the electric problem is a right-handed and orthogonal coordinate, with the origin on the jet axis at the nozzle outlet. Hence, the distance of each point on the surface of the jet to the origin can be calculated by Eq. (18),

$$
\overline{\boldsymbol{r}}=\left(\bar{X}+\bar{R} \bar{Z}_{s} \cos \varphi\right) \boldsymbol{e}_{i}+(\bar{R} \sin \varphi) \boldsymbol{e}_{j}+\left(\bar{Z}-\bar{R} \bar{X}_{s} \cos \varphi\right) \boldsymbol{e}_{k} .
$$

The initial interface profile is divided into $N$ equal triangular elements, located between the nozzle tip and the end of the jet where it is assumed to be truncated. The linear elements are defined by three nodes at each end and a node at the center of the element, which is defined as the calculated node, and Gaussian points are distributed around this node to estimate the surface integral of Eq. (17). A singular element which has source and field points (i.e., $p$ and $q$ ) is subdivided into three elements on a singular point to eliminate the singularity of integrand (17); meanwhile, a correction was done on the Gaussian quadrature over sub-elements. ${ }^{30}$
Similar to all studies in spinning, only a certain length of the jet is analyzed, and the end of the jet is truncated. Hence, the simulated length should be long enough to avoid the occurrence of any numerical errors.

Computing the normal and tangential components of an external electric field in the $3 D$ domain, only components in the $\varphi=0$ plane were extracted according to the governing assumption (slender jet with circular cross section area). In the simulation, the external electric field is obtained by analytically solving the Laplace equation between two cylinders for the electric potential. An external electric field can be achieved in polar coordinates using Eq. (19) which was non-dimensionalized by the magnitude of electric field as Eq. (20),

$$
\begin{aligned}
\overline{\boldsymbol{E}}_{\infty} & =\frac{\left(\bar{X}+\frac{1}{\epsilon}\right) \boldsymbol{e}_{i}+\bar{Z} \boldsymbol{e}_{k}}{\left(\bar{X}+\frac{1}{\epsilon}\right)^{2}+\bar{Z}^{2}}, \\
E_{0} & =-\frac{1}{a} \frac{\Delta \Psi}{\ln \left(\frac{R_{\text {collector }}}{s_{0}}\right)} .
\end{aligned}
$$

Due to the fluid's electrical conductivity used in the current study, $\sigma_{o i}$ tends to zero, and the effect of this parameter in the governing Eqs. (6), (10), and (14) will be neglected. Therefore, the terms including $\sigma_{o i}$ were eliminated from the governing equations for the simulations-since the second fluid surrounding the jet is also air, $\varepsilon_{i o}=\varepsilon_{i}$, in this study.

With the normal and tangential components of the electric field being as described above, the nonlinear equations (6)-(8) can be solved using a Newton method. In this set of equations, the derivatives are discretized using a second-order central difference scheme, and the downstream jet conditions can be obtained from a second order extrapolation of the last internal grid points.

\section{RESULTS AND DISCUSSION}

\section{A. Simulation and comparison with experiment}

In this section, the spinning process through air-sealed electro-centrifugal spinning system is experimentally investigated, and the suggested numerical method for electrocentrifugal spinning will be validated by comparing the simulation and experimental results. The electro-centrifugal spinning setup was first introduced by Dabirian et al. ${ }^{24}$ to produce nanofibers by means of electric and centrifugal forces. Electro-centrifugal spinning suffered from a stream of air surrounding the nozzle. The high-speed airflow caused the liquid jet to dry rapidly, resulting in thicker nanofibers. Thus, Valipouri et al. ${ }^{1}$ enhanced centrifuge spinning by isolating it from the surrounding air by means of a rotating collector. They achieved agreement between the numerical and experimental results in nanofiber production only by centrifugal force. Also, they conducted experiments regarding nanofiber production by electric and centrifugal forces simultaneously. ${ }^{12}$ The setup used in the current study is based on that of Valipouri et al. ${ }^{1,12}$ because there was good agreement between their numerical and experimental findings for centrifugal spinning. This setup uses electric and centrifugal forces, which act as a driving force 
TABLE III. Properties of the utilized Newtonian leaky dielectric fluid (volume fraction of $85 \%$ glycerol and $15 \%$ water) at $20{ }^{\circ} \mathrm{C}$.

\begin{tabular}{lccccc}
\hline \hline$\rho\left(\frac{\mathrm{kg}}{\mathrm{m}^{3}}\right)$ & $\mu($ Pas $)$ & $\gamma\left(\frac{\mathrm{mN}}{\mathrm{m}}\right)$ & $\varepsilon(-)$ & $\sigma\left(\frac{\mu \mathrm{S}}{\mathrm{m}}\right)$ & $a(\mathrm{~mm})$ \\
\hline 1228 & 0.138 & 64 & 40 & 1.28 & 0.35 \\
\hline \hline
\end{tabular}

to draw the fluid out of the nozzle-requiring a higher rotational speed - and the flow rate is calculated by averaging the time needed for a specific amount of liquid to discharge.

Hohman et al. ${ }^{3}$ figured out that the protrusion length of the nozzle through the capacitor plate has a noticeable effect on the non-uniformity of the electric field in the vicinity of the nozzle in an electro-spinning process. To explain this finding, they propounded the existence of a fringe field near the nozzle tip. Accordingly, in the present study, certain experiments were prepared using a Newtonian leaky dielectric fluid (volume fraction of $85 \%$ glycerol and $15 \%$ water-with the properties listed in Table III) and different setups to induce the electric field near the nozzle.

The initial jet development in the scope of air-sealed electro-centrifugal spinning was photographed using a Canon EOS $6 D$ DSLR camera, a $200 \mathrm{~mm}$ f/4d Micro Nikkor lens, and a Canon 430 EXII speed light. The electric field was formed by a high voltage power supply with a maximum nominal voltage of $30 \mathrm{kV}$ and two aluminum electrodes. Additionally, the solution viscosity, electrical conductivity, and the surface tension were measured by a $D V-I I+$ Pro viscometer, an $E U$ 3540 conductivity meter, a DCAT 11 surface tension instrument, and a Libror AEU-210 balance measurement device, respectively.

In this study, the effect of electrode configuration on the electric field around the nozzle tip was experimentally studied through a plate-plate geometry, with the nozzle tip protruding from the top of a capacitor plate, and also through a point-plate geometry, with one of the electrodes directly connected to the nozzle. Moreover, the radius of the inner cylinder and the spinning distances were adjusted to $5.3 \mathrm{~cm}$ and $8 \mathrm{~cm}$, respectively. Corresponding dimensionless numbers to the conditions of spinning experiments are listed in Table IV.

TABLE IV. Dimensionless numbers corresponding to the conditions of spinning experiments.

\begin{tabular}{|c|c|c|c|c|c|c|}
\hline & $W e \times 10^{3}$ & $\operatorname{Re} \times 10^{2}$ & $F r \times 10^{2}$ & $R b \times 10$ & $\left(\frac{\beta}{E_{0}^{2}}\right)\left(\frac{\Delta \Psi}{R_{\text {collector }}-s_{0}}\right)$ & $\epsilon \times 10^{3}$ \\
\hline \multicolumn{7}{|c|}{ Point-plate geometry (Fig. 3) } \\
\hline$a$ & 1.6 & 4.9 & 7.1 & 3.0 & 0.7 & 4.6 \\
\hline$b$ & 0.9 & 3.6 & 4.0 & 2.2 & 2.0 & 4.6 \\
\hline$c$ & 0.2 & 1.6 & 0.8 & 1.3 & 16.1 & 4.6 \\
\hline \multicolumn{7}{|c|}{ Plate-plate geometry (Fig. 4) } \\
\hline & & & & & $\beta \times 10^{-6}$ & \\
\hline$a$ & 0.9 & 3.6 & 4.0 & 2.2 & 0.9 & 3.7 \\
\hline$b$ & 2.0 & 5.4 & 8.7 & 3.8 & 0.4 & 3.7 \\
\hline$c$ & 0.7 & 3.1 & 2.9 & 2.5 & 1.2 & 3.7 \\
\hline$d$ & 1.0 & 3.8 & 4.3 & 2.3 & 0.6 & 4.6 \\
\hline$e$ & 1.6 & 4.9 & 7.1 & 3.4 & 0.8 & 4.0 \\
\hline
\end{tabular}

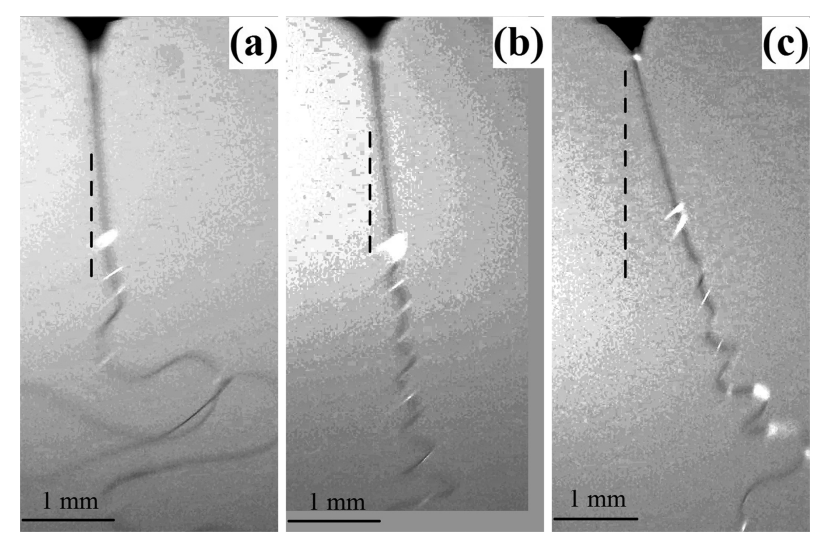

FIG. 3. Electro-centrifugal spinning process for point-plate geometry and a constant distance of $5.7 \mathrm{~cm}$ between the nozzle tip and the collector, and operating parameters [rotational speed $(\mathrm{rpm})$, flow rate $(\mathrm{ml} / \mathrm{min})$, and applied potential differences (kV)] are (a) $(1440,0.36,9)$, (b) $(1440,0.27,11)$, and (c) $(1080,0.12,14)$.

The experimental results for the point-plate geometry are illustrated in Fig. 3. As can be seen in Figs. 3(a) and 3(b) for a constant rotational speed, increasing the applied potential difference changed the jet instability from whipping into spiral instabilities. In addition, the jet trajectory deviated slightly more from the straight path. It has been previously found that the centrifugal force without the presence of the electric field could not create whipping instability. ${ }^{1,2}$ The possible reasons for whipping or spiraling instability could be the nonuniformity of the electric field in the vicinity of the nozzle. Also, Fig. 3(c) illustrates that jet deviation increased with decreasing rotational speed.

The experimental results for the plate-plate geometry with the metal nozzle protruding from the capacitor plate are illustrated in Fig. 4. Figures 4(a) and 4(d), as well as Figs. 4(b) and 4(e), show that increasing the potential difference (under a constant rotational speed) will lead to a more pronounced deviation of the jet trajectory. The jet starts to exhibit instability, but this is suppressed by reducing the protrusion length of the nozzle. By comparing Figs. 3(c) and 4(c), it can be observed that, for the same operating parameters, there is an increase in the jet trajectory's deviation from a straight line, while the instabilities decrease using plate-plate geometry. As a result, it can be stated that a capacitor plate attached to the nozzle will reduce the non-uniformity of the electric field. In addition, to diminish electric field irregularities close to the nozzle by the capacitor plate, the protrusion length of the nozzle is also an important parameter in jet instabilities, similar to the electro-spinning process. ${ }^{3}$

The difference between these two mechanisms is the lifting of the electric field's unevenness near the nozzle for plate-plate configuration. To make a clear comparison between point-plate and plate-plate geometries, jet trajectories obtained from Figs. 3 and 4 were illustrated in Fig. 5.

By comparing point-plate and plate-plate geometries, it can be concluded that the metal plate attached to the nozzle has decreased the electric field irregularities as the jet instabilities occurred at a longer distance from the nozzle. Furthermore, the jet's stability is increased by increasing the uniformity of the electric field by a plate-plate mechanism. 

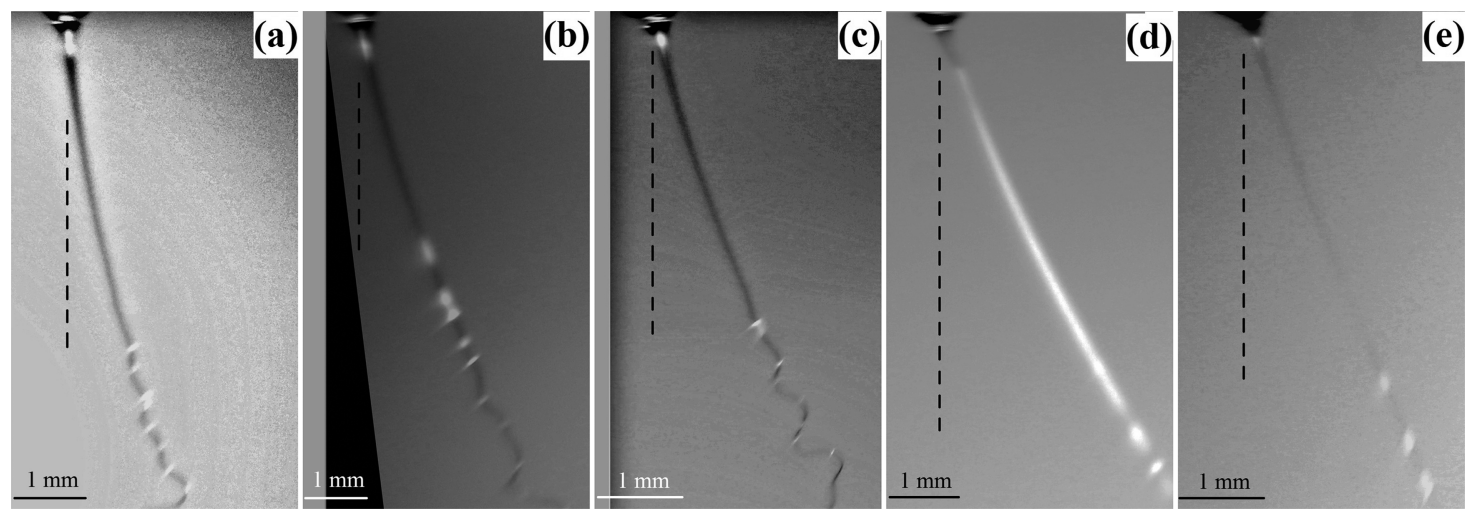

FIG. 4. Electro-centrifugal spinning process for plate-plate geometry, and operational parameters [rotational speed (rpm), flow rate ( $\mathrm{ml} / \mathrm{min})$, applied potential differences $(\mathrm{kV})$, and nozzle protrusion length (mm)] are (a) $(1440,0.27,15,42)$, (b) $(1260,0.40,15,42),(\mathrm{c})(1080,0.23,15,42),(\mathrm{d})(1440,0.28,22,23)$, and (e) $(1260,0.36,25,34)$.
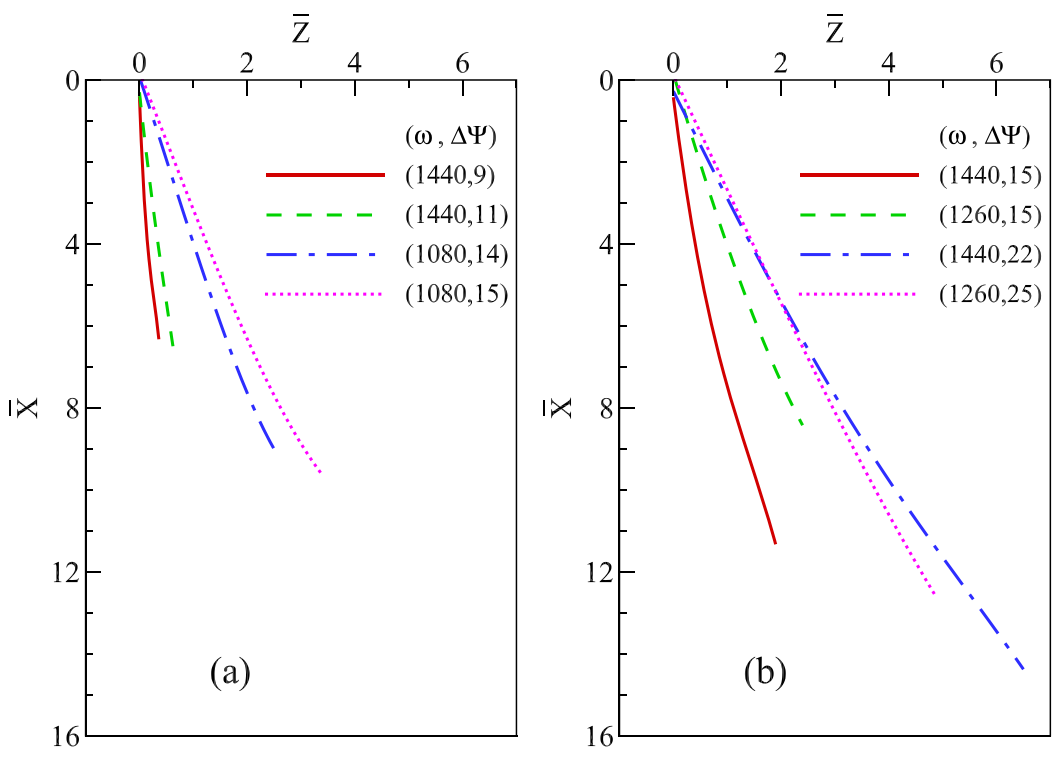

FIG. 5. Quantitative comparison between experimental results of point-plate (a) and plate-plate (b) geometries. Moreover, there is a comparison between point-plate and plate-plate geometry result in (a).

In our numerical model for the electrified jet, we assumed that the position of the central axis of the jet is not affected by the small perturbations. However, it is necessary to provide experimental conditions in line with the numerical model to correctly compare these results. Experimental results of Fig. 4 show that the plate-plate configuration with the nozzle protruding from a metal plate is more consistent with the assumptions of the present simulation. Moreover, experimental evidence showed that the electric field generated by nozzle protrusion length of $23 \mathrm{~mm}$ is closer to the numerical model assumptions used in this study [Fig. 4(d)].

In order to validate this proposition, a comparison was conducted between our numerical model results and experimental results. Figure 6 shows a comparison of the experimental parameters of Table III and the corresponding dimensionless numbers used in numerical simulations, as listed in Table V. As shown by the figure, good agreement is achieved for the proposed electrode configuration (i.e., plate-plate geometry) and the static model for surface charges.
To bring the numerical model assumptions closer to the physical conditions of the experiment, the effect of gravity forces is also included to the governing equations by adding $\bar{Z}_{s} / F r$ and $\bar{X}_{s} / F r$ terms to Eqs. (6) and (7), respectively.

\section{B. Numerical study on electro-centrifugal spinning}

In spinning processes, it is quite popular to use electric and centrifugal forces separately-but there have been no recent attempts to carry out numerical investigations and experiments on combinations of the two aspects. Therefore, we worked on a numerical comparison of centrifugal spinning $(\Delta \Psi=0)$ and electro-centrifugal spinning $(\Delta \Psi \neq 0)$ for a fluid with specific physical properties as listed in Table VI. Also, Table VII shows the dimensionless numbers used in the numerical simulation.

Figure 7 depicts the effects of the electric potential difference on a steady jet trajectory in the $\bar{X}-\bar{Z}$ plane and on the jet radius along the axis at different rotational speeds. As can be seen, increasing the applied potential difference at a constant rotational speed increases the deviation of the jet trajectory 

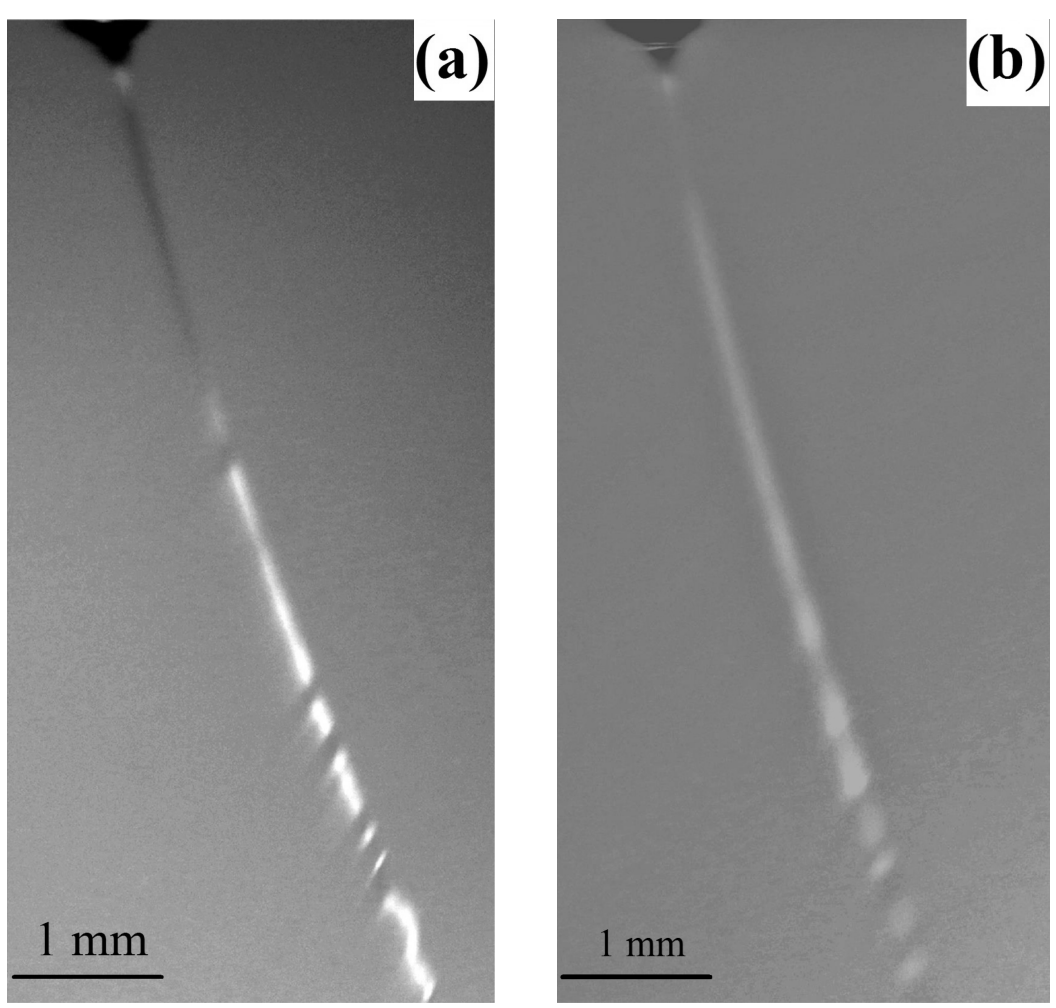

(b)

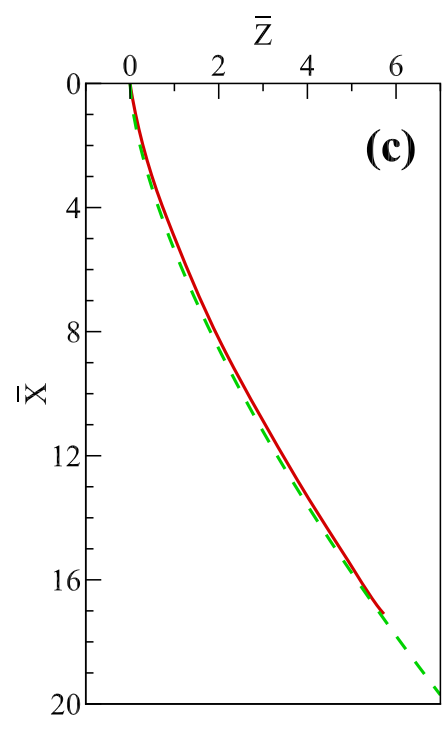

$---{ }_{-}$Numerical simulation

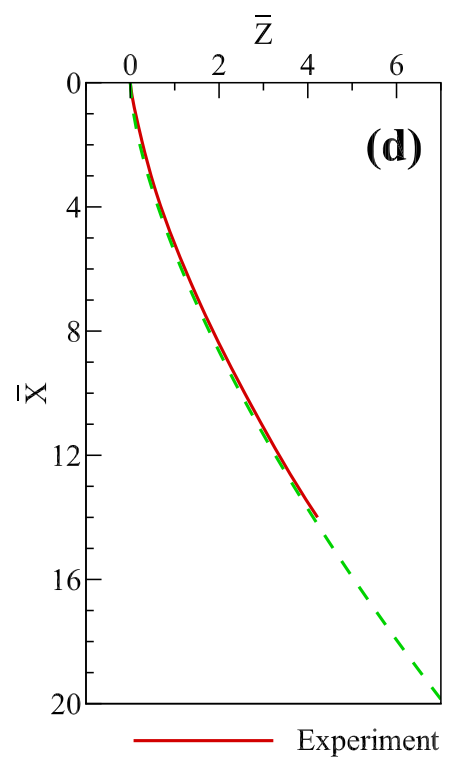

FIG. 6. Comparison of numerical results (dashed line) and experimental results (solid line) for electrocentrifugal jet behavior. Operational parameters are [rotational speed (rpm), flow rate $(\mathrm{ml} / \mathrm{min})$, and applied potential differences $(\mathrm{kV})]$ (a) $(1350,0.37,25)$ and (b) $(1440$, $0.30,22)$. from a straight line in the $\bar{X}-\bar{Z}$ plane-which is in agreement with the experimental results shown in Figs. 3(a) and 3(b). However, increasing the rotational speed will reduce the influence of the applied potential difference on the jet behavior. Moreover, it is predicted that the jet thins out faster as it moves away from the spinneret by increasing the applied

TABLE V. Dimensionless numbers based on the parameters in Table III.

\begin{tabular}{ccccccc}
\hline \hline & $W e \times 10^{3}$ & $R e \times 10^{2}$ & $F r \times 10^{2}$ & $R b \times 10$ & $\beta \times 10^{-5}$ & $\epsilon \times 10^{3}$ \\
\hline$c$ & 1.1 & 4.0 & 4.9 & 2.6 & 7.0 & 4.6 \\
$d$ & 1.7 & 5.0 & 7.5 & 3.0 & 3.5 & 4.6 \\
\hline \hline
\end{tabular}

potential difference. In fact, the increase of the applied potential difference leads to an increase in the jet driving force. However, the effect of the applied potential difference is suppressed at a high rotational speed.

It can be concluded that, at a high rotational speed, the applied potential difference has no significant effect on the jet behavior (i.e., trajectory and radius profile) as shown by the experimental results of Dabirian et al. ${ }^{25}$

\section{Parameters study}

In order to analyze the effects of fluid physical properties, geometrical parameters, and flow characteristics on the behavior of the electrified jet, we studied the effects of various 
TABLE VI. Fluid properties and geometrical characteristics.

\begin{tabular}{ccccccccc}
\hline \hline$\rho\left(\frac{\mathrm{kg}}{\mathrm{m}^{3}}\right)$ & $\mu(\mathrm{Pa} \mathrm{s})$ & $\gamma\left(\frac{\mathrm{mN}}{\mathrm{m}}\right)$ & $\varepsilon(-)$ & $\sigma\left(\frac{\mu \mathrm{S}}{\mathrm{m}}\right)$ & $Q\left(\frac{\mathrm{ml}}{\mathrm{min}}\right)$ & $a(\mathrm{~mm})$ & $s_{0}(\mathrm{~cm})$ & $R_{\text {collector }}(\mathrm{cm})$ \\
\hline 1260 & 1.67 & 64 & 42.5 & 1.0 & 0.5 & 0.35 & 5.3 & 13.3 \\
\hline \hline
\end{tabular}

TABLE VII. Dimensionless numbers used for the numerical simulation of electro-centrifugal spinning based on the parameters in Table VI.

\begin{tabular}{cccccccccc}
\hline \hline & \multicolumn{1}{c}{$\Delta \Psi(\mathrm{kV})$} & \multicolumn{6}{c}{$\omega(\mathrm{rpm})$} \\
\hline 15 & 25 & 35 & & & 500 & 1000 & 1500 \\
& $\beta \times 10^{-5}$ & & $W e \times 10^{3}$ & $\operatorname{Re} \times 10^{3}$ & $F r$ & $R b \times 10$ & & $\epsilon \times 10^{3}$ \\
0.7 & 1.8 & 3.6 & 1.1 & 4.0 & 0 & 4.1 & 2.0 & 1.4 & 9.4 \\
\hline \hline
\end{tabular}

dimensionless numbers. Table VIII represents the main dimensionless numbers considered in the simulations. The effect of dimensionless numbers can be investigated by changing one of the numbers in a specific range while keeping others constant. The simulation results are illustrated in five distinct diagrams in Fig. 8 and will be discussed in more detail in the following.

As can be seen in Fig. 8, decreasing the We number or increasing $R e, R b$, and $\beta$ numbers causes an enhanced jet curvature or jet deviation trajectory from the $\bar{Z}$ axis in the $\bar{X}-\bar{Z}$ plane.

In electro-centrifugal spinning, centrifugal and electric forces act against viscose and surface tension forces. Jet formation means that centrifugal and electric forces prevail surface tension. Moreover, any shape change-such as jet thinning or jet trajectory deviation in the $\bar{X}-\bar{Z}$ plane-means that
TABLE VIII. Dimensionless numbers used in the parameters study.

\begin{tabular}{ccccccc}
\hline \hline$W e \times 10^{3}$ & $R e \times 10^{3}$ & $F r$ & $R b \times 10$ & $\beta \times 10^{-4}$ & $\epsilon \times 10^{3}$ & $\varepsilon_{i o}$ \\
\hline 1 & 10 & 0 & 5 & 3 & 10 & 15 \\
\hline \hline
\end{tabular}

two previously mentioned forces dominate the viscose force. Therefore, increasing We, Re, and $\beta$ numbers or decreasing the $R b$ number will result in a more pronounced jet thinning (Fig. 8).

In Fig. 8, jet trajectory in the $\bar{X}-\bar{Z}$ plane and radius profile along the jet axis for different $\epsilon$ number are presented. This dimensionless number shows the ratio of nozzle radius to distance between the rotation center and the nozzle tip. By reducing this ratio, jet deviation trajectory from the $\bar{Z}$ axis in the $\bar{X}-\bar{Z}$ plane is decreased and jet elongation is increased.

It can also be concluded that-unlike electro-spinning, where the jet profile at the nozzle exit is spherical for a specific range of dimensionless numbers-the jet profile in electro-centrifugal spinning has a conical shape. This indicates that, due to the presence of the centrifugal force, the effects of surface tension are smaller in this process.
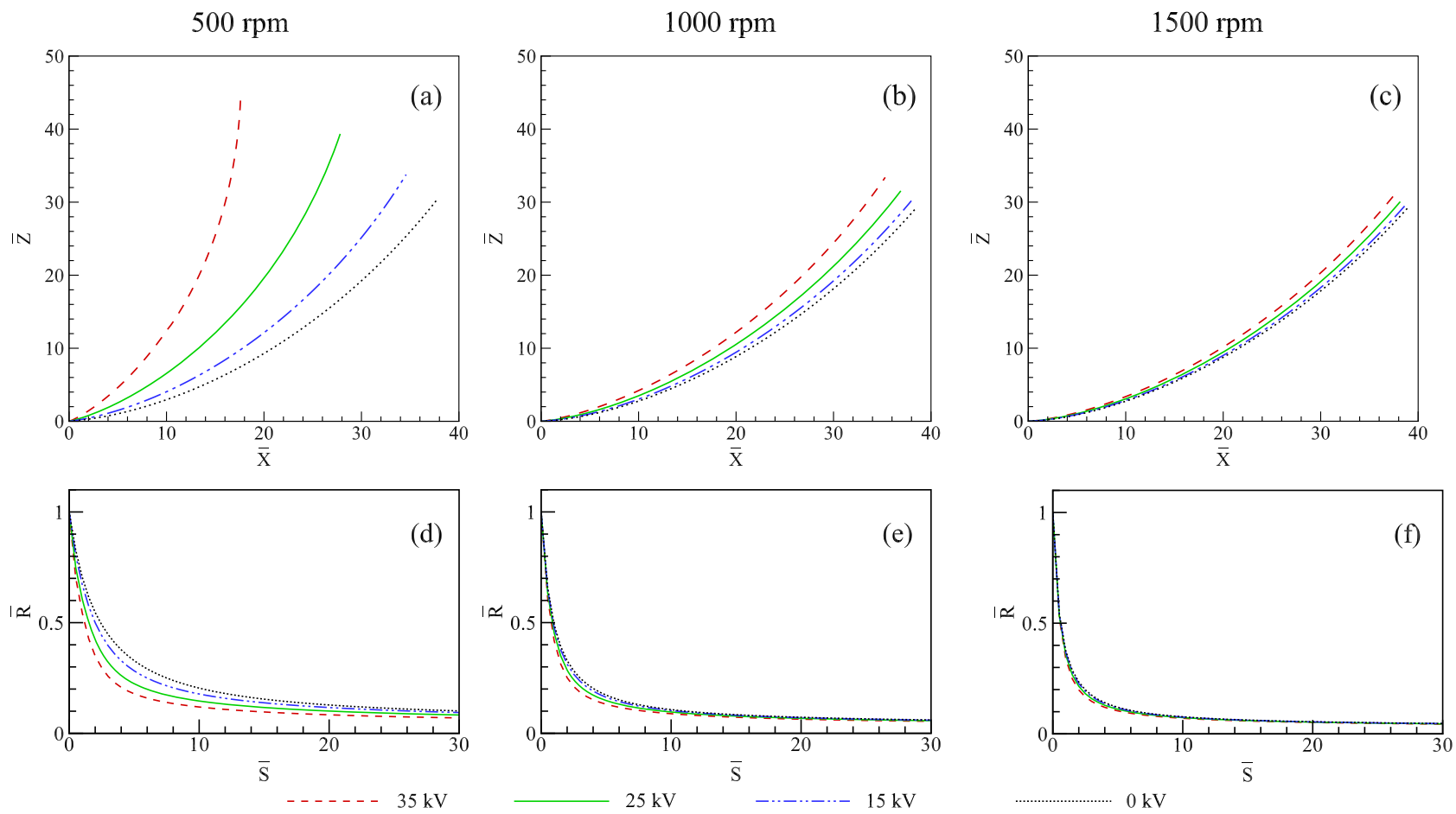

FIG. 7. Effect of the electric potential difference at different rotational speeds on the jet trajectory in the $\bar{X}-\bar{Z}$ plane (first row) and the jet radius profile along the jet axis (second row). 

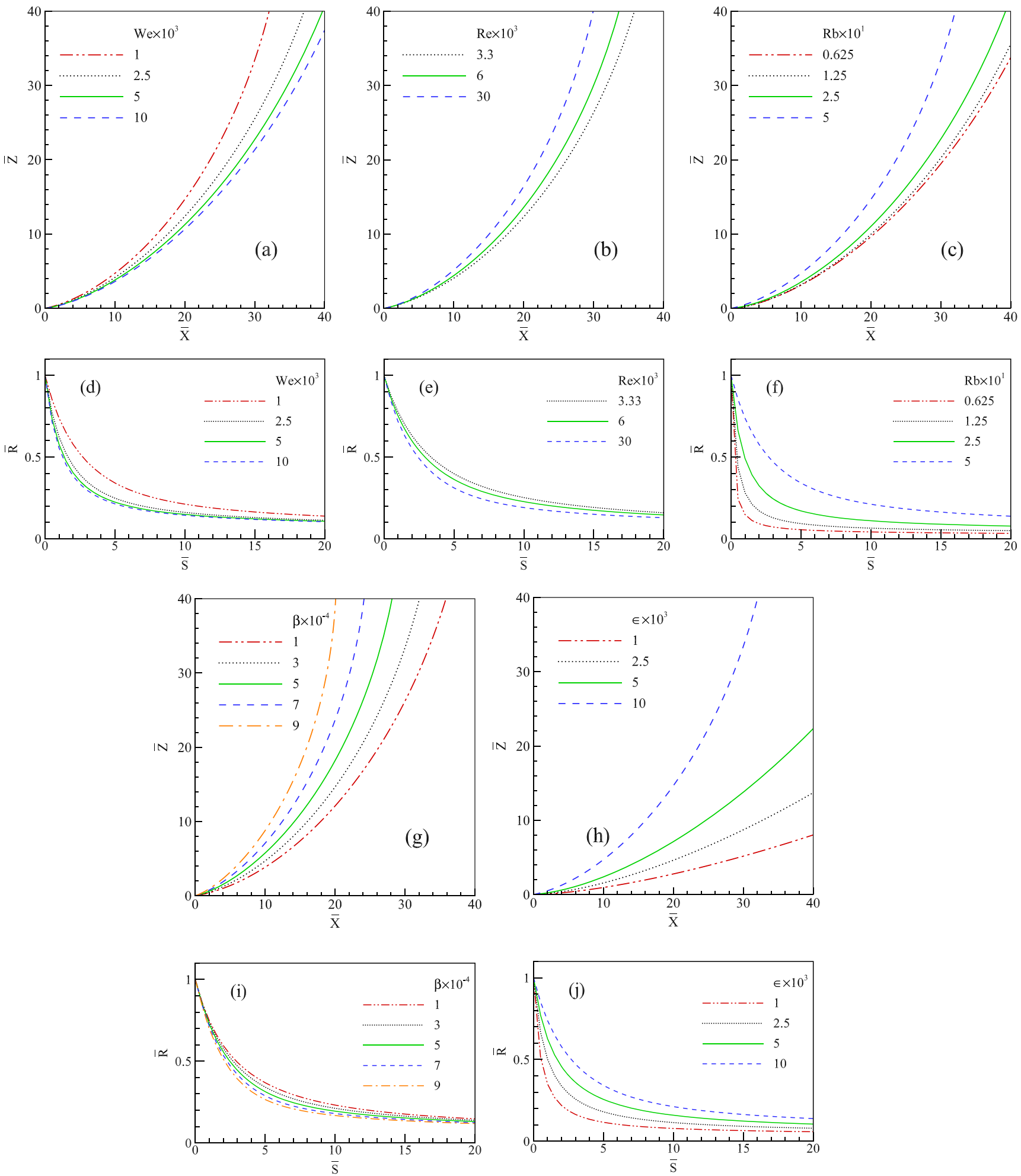

FIG. 8. Effects of dimensionless numbers $W e, R e, R b, \beta$, and $\epsilon$ on the jet trajectory and jet radius profile.

\section{CONCLUSION}

In this study, a numerical model was suggested for predicting the behavior of a Newtonian leaky dielectric rotary jet in an external electric field. The electro-hydrodynamic equations include continuity, momentum, and electric potential equations. In order to validate the numerical model, a comparison was carried out between the numerical results and the experimental results of the present study. This comparison indicated that very good agreement exists between the model results and the experiment.

To match the model assumptions with the real physical conditions in the experiment, several electrode geometries were investigated in the experimental setup, including a direct 
connection between the high voltage source and the nozzle (i.e., point-plate geometry) or a metal plate from which the nozzle protrudes (i.e., plate-plate geometry). The difference between these two mechanisms lies in a reduction of the electric field irregularities in the vicinity of the nozzle. It was observed that the protrusion length of the nozzle noticeably affects the electric field near the nozzle. Moreover, empirical results showed that the mechanism that matches the numerical formulation of the current study is the one in which the high voltage source touches the metal plate that is attached to the nozzle.

Centrifugal force is the reason for the jet deviation from a straight path to a curved path. At the high rotational speed, the centrifugal force exceeds the electric forces and electrocentrifugal spinning becomes centrifugal spinning. An analysis of the effects of dimensionless numbers on the jet behavior indicates that increasing the $R e, R b, \beta$, and $\epsilon$ numbers and reducing the We number will lead to an increased jet deviation trajectory. Further, increasing $W e, R e$, and $\beta$-as well as reducing $R b$ and $\epsilon$-leads to a more pronounced jet stretching.

${ }^{1}$ A. Valipouri, S. A. H. Ravandi, A. Pishevar, and E. I. Părău, "Experimental and numerical study on isolated and non-isolated jet behavior through centrifuge spinning system," Int. J. Multiphase Flow 69, 93-101 (2015).

${ }^{2}$ S. Padron, A. Fuentes, D. Caruntu, and K. Lozano, "Experimental study of nanofiber production through forcespinning," J. Appl. Phys. 113(2), 024318 (2013).

${ }^{3}$ M. M. Hohman, M. Shin, G. Rutledge, and M. P. Brenner, "Electrospinning and electrically forced jets. II. Applications,” Phys.Fluids 13(8), 2221-2236 (2001).

${ }^{4}$ C. P. Carroll and Y. L. Joo, "Electrospinning of viscoelastic Boger fluids: Modeling and experiments," Phys. Fluids 18, 053102 (2006).

${ }^{5}$ C. J. Ellison, A. Phatak, D. W. Giles, C. W. Macosko, and F. S. Bates, "Melt blown nanofibers: Fiber diameter distributions and onset of fiber breakup," Polymer 48, 3306-3316 (2007).

${ }^{6}$ P. X. Ma and R. Zhang, "Synthetic nano-scale fibrous extracellular matrix," J. Biomed. Mater. Res. 46, 60-72 (1999).

${ }^{7}$ G. Liu, J. Ding, L. Qiao, A. Guo, B. P. Dymov, J. T. Gleeson, T. Hashimoto, and K. Saijo, "Polystyrene-block-poly (2-cinnamoylethyl methacrylate) nanofibers-Preparation, characterization, and liquid crystalline properties," Chem. - Eur. J. 5, 2740-2749 (1999).

${ }^{8}$ L. Feng, S. Li, H. Li, J. Zhai, Y. Song, L. Jiang, and D. Zhu, "Superhydrophobic surface of aligned polyacrylonitrile nanofibers," Angew. Chem. 114, 1269-1271 (2002).

${ }^{9}$ C. R. Martin, "Membrane-based synthesis of nanomaterials," Chem. Mater. 8, 1739-1746 (1996).

${ }^{10}$ K. Sarkar, C. Gomez, S. Zambrano, M. Ramirez, E. de Hoyos, H. Vasquez, and K. Lozano, "Electrospinning to forcespinning TM," Mater. Today 13(11), 12-14 (2010).
${ }^{11}$ A. L. Andrady, D. S. Ensor, and R. J. Newsome, "Electrospinning of fibers using a rotatable spray head," U.S. patent 7,134,857 (14 November 2006).

${ }^{12}$ A. Valipouri, S. A. H. Ravandi, and A. Pishevar, "Optimization of the parameters involved in fabrication of solid state polymerized polyamide (SSP PA66) nanofibers via an enhanced electro-centrifuge spinning,' J. Ind. Text. 45(3), 368-386 (2015).

${ }^{13}$ J.-M. Vanden-Broeck and J. B. Keller, "Jets rising and falling under gravity," J. Fluid Mech. 124, 335-345 (1982).

${ }^{14}$ V. Entov and A. Yarin, "The dynamics of thin liquid jets in air," J. Fluid Mech. 140, 91-111 (1984).

${ }^{15}$ F. Dias and J.-M. Vanden-Broeck, "Flows emerging from a nozzle and falling under gravity," J. Fluid Mech. 213, 465-477 (1990).

${ }^{16}$ D. S. Finnicum, S. J. Weinstein, and K. J. Ruschak, "The effect of applied pressure on the shape of a two-dimensional liquid curtain falling under the influence of gravity," J. Fluid Mech. 255, 647-665 (1993).

${ }^{17}$ L. Cummings and P. Howell, "On the evolution of non-axisymmetric viscous fibres with surface tension, inertia and gravity,” J. Fluid Mech. 389, 361-389 (1999).

${ }^{18}$ D. H. Reneker and A. L. Yarin, "Electrospinning jets and polymer nanofibers," Polymer 49(10), 2387-2425 (2008).

${ }^{19}$ I. Wallwork, S. Decent, A. King, and R. Schulkes, "The trajectory and stability of a spiralling liquid jet. Part 1 . Inviscid theory," J. Fluid Mech. 459, 43-65 (2002).

${ }^{20}$ S. Decent, A. King, and I. Wallwork, "Free jets spun from a prilling tower," J. Eng. Math. 42(3), 265-282 (2002).

${ }^{21}$ D. Wong, M. Simmons, S. Decent, E. Parau, and A. King, "Break-up dynamics and drop size distributions created from spiralling liquid jets," Int. J. Multiphase Flow 30(5), 499-520 (2004).

${ }^{22}$ E. I. Părău, S. P. Decent, M. J. H. Simmons, D. C. Y. Wong, and A. C. King, "Nonlinear viscous liquid jets from a rotating orifice," J. Eng. Math. 57, 159-179 (2007)

${ }^{23}$ F. Dabirian, S. Sarkeshik, and A. Kianiha, "Production of uniaxially aligned nanofibers using a modified electrospinning method: Rotating jet," Curr. Nanosci. 5(3), 318-323 (2009).

${ }^{24}$ F. Dabirian, S. Hosseini Ravandi, and A. Pishevar, "Investigation of parameters affecting PAN nanofiber production using electrical and centrifugal forces as a novel method," Curr. Nanosci. 6(5), 545-552 (2010).

${ }^{25}$ F. Dabirian, S. H. Ravandi, and A. Pishevar, "The effects of operating parameters on the fabrication of polyacrylonitrile nanofibers in electro-centrifuge spinning," Fibers Polym. 14(9), 1497-1504 (2013).

${ }^{26}$ E. Lac and G. M. Homsy, "Axisymmetric deformation and stability of a viscous drop in a steady electric field," J. Fluid Mech. 590, 239-264 (2007).

${ }^{27}$ D. A. Saville, "Electrohydrodynamics: The Taylor-Melcher leaky dielectric model," Annu. Rev. Fluid Mech. 29, 27-64 (1997).

${ }^{28}$ H. Paknemat, A. R. Pishevar, and P. Pournaderi, "Numerical simulation of drop deformations and breakup modes caused by direct current electric fields," Phys. Fluids 24, 102101 (2012).

${ }^{29}$ M. Kang, R. P. Fedkiw, and X.-D. Liu, “A boundary condition capturing method for multiphase incompressible flow,” J. Sci. Comput. 15, 323-360 (2000).

${ }^{30}$ S. M. Kirkup, "Solution of Exterior Acoustic Problems by the Boundary Element Method," Ph.D. thesis (Brighton Polytechnic, Brighton, UK, 1989). 